\title{
Metabolisches Syndrom
}

\section{Liebe Leserinnen, liebe Leser,}

das gemeinsame Auftreten der Stoffwechselstörungen Adipositas, Hypertonie, Hyperglykämie und Dyslipidämie, v.a. Hypertriglyceridämie, wird als metabolisches Syndrom bezeichnet. Der Vergleich der Prävalenzen des metabolischen Syndroms in verschiedenen Ländern ist durch unterschiedliche Definitionen erschwert. In den zurückliegenden Jahren gab es immer wieder neue Definitionen, $z$. B.

- 1998 von der WHO,

- 1999 von der EGI (European Group for the Study of Insulin Resistance),

- 2001 von dem NCEP (National Cholesterol Education Program),

- 2002 von dem ACE (American College of Endocrinology),

- 2004 von der AHA (American Heart Association) und

- 2005 von der IDF (International Diabetes Federation).

Bewertungskriterien sind der Hüftumfang als Maß der abdominalen Fettansammlung, ein erhöhter Blutdruck bzw. eine antihypertensive Therapie (IDF), erhöhter Nüchtern-Blutzuckerspiegel bzw. Blutzucker senkende Medikation (IDF), erhöhte Triglyceride bzw. Triglycerid senkende Medikation (IDF) und erniedrigte HDL-Spiegel. Die strengsten Kriterien werden nach der IDF-Definition angelegt.

Die Prävalenz des metabolischen Syndroms in Deutschland liegt laut GEM-
CAS-Studie (German Metabolic and Cardiovascular Risk Project) von 2007 je nach Definition zwischen 18,7\% (NECP) und 30,7\% (IDF). Sie steigt mit zunehmendem Lebensalter bis zum ca. 75. Lebensjahr an und fällt in höherem Lebensalter ganz leicht ab. Bis zum ca. 70.Lebensjahr sind Männer häufiger betroffen als Frauen. Vergleichbare Ergebnisse finden sich für die meisten europäischen Länder sowie die USA. Auffällig ist eine deutliche Korrelation zwischen Prävalenz des metabolischen Syndroms und der Schulbildung. Dieser Unterschied liegt je nach Altersgruppe bei mehr als $100 \%$ ! D. h. je geringer die Schulbildung, desto höher das Risiko für ein metabolisches Syndrom.

Auch die norwegische HUNT-2-Studie zeigt ein ähnliches Ergebnis mit einer Gesamtprävalenz von 29,0\% für Männer und 30,3\% für Frauen (nach IDF). Diese Zahlen sind alarmierend, da schon ca. $10 \%$ der jungen Menschen zwischen 20 und 30 unter dem metabolischen Syndrom leiden. Bei dieser Altersgruppe steht das Übergewicht an erster Stelle der Risikoparameter. Der moderne Lebensstil mit einem ausgeprägten Bewegungsmangel sowie hyperkalorischer Fehlernährung steht am Anfang einer Entwicklung, die immer mehr Menschen betrifft und verantwortlich ist für den dramatischen Anstieg von Diabetes Typ 2 und seinen Folgeerkrankungen wie Arteriosklero- se, Neuropathien, Niereninsuffizienz, Retinopathien mit Erblindung etc. Übergewicht ist dabei ein zentraler Ausgangspunkt. Das Fettgewebe ist stoffwechselaktiv und u.a. Bildungsort proinflammatorischer Zytokine, die über verschiedene Mechanismen den Glucose- und Fettstoffwechsel verändern und zur Entwicklung eines Diabetes Typ 2 beitragen. 80\% der Typ-2-Diabetiker sind übergewichtig. Die Tatsache, dass $20 \%$ der Schulkinder in Deutschland übergewichtig sind und ca. 8\% die Kriterien einer Adipositas erfüllen, erfordert umgehendes Handeln und dramatische Veränderungen im Bereich Bewegung und Ernährung schon im Kindesalter.

Wir wünschen Ihnen viel Spaß mit der aktuellen Ausgabe der OM und viel Erfolg bei der Umsetzung neu gewonnener Erkenntnisse.

Ihre Herausgeber
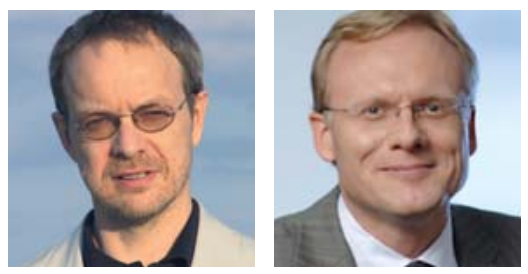

Dr. med. Hans-Peter Friedrichsen

Apotheker Uwe Gröber 